\title{
The rheology of a dilute suspension of Brownian dipolar spheroids in a simple shear flow under the action of an external force
}

\author{
K. Asokan \\ School of Technology and Applied Sciences, Mahatma Gandhi University, Regional Center, \\ Pathanamthitta-689 645, India \\ T. R. Ramamohana) \\ C-MMACS, Bangalore-560 037, India
}

(Received 11 April 2003; accepted 30 October 2003; published online 8 January 2004)

\begin{abstract}
The effect of rotational Brownian motion on the rheology of a dilute suspension of dipolar spheroids in a simple shear flow under the action of an external force field, is investigated through a generalized Langevin equation approach. The force field is assumed to be either constant or periodic. In the case of constant external fields earlier results in the literature are reproduced, while for the case of periodic forcing certain parametric regimes corresponding to weak Brownian diffusion are identified where the rheological parameters evolve chaotically and settle onto a low dimensional attractor. The response of the system to variations in the strengths of the force field and diffusion is also analyzed through numerical experiments. These results correspond to the region of weak Brownian motion where usual methods render the problem intractable. (C) 2004 American Institute of Physics. [DOI: 10.1063/1.1635821]
\end{abstract}

\section{INTRODUCTION}

The study of suspensions of dipolar particles subjected to an external rotational torque has important engineering applications, such as controlling the processing of fiber composites, magnetofluidization, ${ }^{1}$ magnetostriction of ferromagnetic particle suspensions, ${ }^{2}$ characterization of magnetorheological suspensions, ${ }^{3}$ etc. Ferrofluids, which contain small single-domain particles in a nonmagnetic solvent, are an important example of dipolar suspensions. They have many industrial applications such as in rotary seals, inertia dampers, magnetic domain detection, and biomedical uses like the concentration of drugs at body sites. ${ }^{4,5}$ When brought under the influence of a magnetic field, the particles in a ferrofluid experience additional torques due to the external field tending to align the particle dipole axis along the field direction, and this affects their macroscopic behavior substantially. In turn, the development of models for the dynamics and bulk properties of such suspensions is useful in designing new suspensions with desired properties, controlling and testing the quality of suspensions, improving the processing conditions of heterogeneous media, ${ }^{4}$ etc. As an example of natural phenomena analogous to the above, Pedley and Kessler ${ }^{6,7}$ refer to certain bacteria containing magnetic dipoles and various species of algae possessing an asymmetric internal mass distribution whose swimming directions are affected by gravity. All of these systems can be modeled by the response of a permanent dipole exposed to an external field.

The effect of fluid microstructure on macroscopic suspension properties is usually measured by the stress tensor of the suspension. In this paper we compute the stress tensor in

a) Author to whom all correspondence should be addressed. Electronic mail: trr@cmmacs.ernet.in a sheared suspension of dipolar spheroids under the action of hydrodynamic forces, Brownian rotations and an external force field (which may be constant or periodic). The apparent viscosities and the first and second normal stress differences are important stress components of a suspension that measure in some sense the collective behavior of all the particles in the suspension. These can be expressed in terms of appropriate particle orientation averages, and the computation of these averages is an important step in any investigation of bulk suspension properties. This is generally made difficult by the complicated manner in which the torques due to shear, Brownian forces, and external field tend to orient and disorient the particles. While suspensions in the absence of external forcing have been extensively studied, only a relatively few investigations are available for the case of dipolar particles, and most of these are restricted to the limit of weak shear. The earliest of these studies were of Hall and Busenberg $^{8}$ and Brenner, ${ }^{9}$ concerning dilute suspensions of dipolar non-Brownian spheres. Brenner and Weissman ${ }^{10}$ extended these studies incorporating Brownian diffusion effects and obtained results mainly for particles that are spheres or near-spheres. Further extensions of these results, including the effect of the relative strengths of shear, Brownian diffusion and external force on the particle dynamics, and rheology, are available, ${ }^{11-13}$ but most of these apply to the limit of weak shear. Strand and $\mathrm{Kim}^{4}$ considered dilute dipolar suspensions for a wider range of shear and diffusion parameters, and analyzed the rheology for various orientations of the external force. Almog and Frankel ${ }^{14,15}$ present several results for the long term behavior of the orientation distribution function of dipolar particles in shear flow, and discuss the implications of these results for the rheology of Brownian dipolar axisymmetric particles. These results are not re- 
stricted to the limit of weak shear effects, unlike the previous studies.

Ramamohan et al. introduced a class of problems, viz., the dynamics and rheology of periodically forced particles in a simple shear flow, and obtained results that were theoretically interesting and had potential technical applications. ${ }^{16-22}$ Kumar et al. ${ }^{17}$ and Kumar and Ramamohan ${ }^{18}$ have demonstrated that both the dynamics and the rheology of such suspensions can be chaotic in certain parametric regimes. The dynamics in this regime is featurerich and presents a new class-I intermittency route to chaos ${ }^{20}$ while the chaotic response of the rheology has important implications for certain aspects of chaos theory such as spatiotemporal chaos. ${ }^{22}$ All the above results were limited to the case of zero or negligible Brownian motion and were obtained by a direct simulation of the equations of motion for particle orientations. In the present work, we expand the scope of their analysis to more realistic suspensions by including the effects of rotary Brownian motion on the bulk suspension properties and demonstrate that the fluctuations in the bulk properties can still be chaotic, for certain ranges of parameters, when the Brownian diffusion is weak.

In the presence of Brownian diffusion, the particle orientations may be considered a stochastic process and may be modeled either through a Fokker-Planck (diffusion) equation formalism or through a Langevin equation formalism. In the first approach the system is studied through a partial differential equation governing the time and space variation of an appropriate density function for the stochastic variable, called the orientation distribution function (ODF) in the present case. In the second method the system is modeled through a set of stochastic differential equations, called Langevin equations, governing the evolution in time of the stochastic variables. Most of the literature in this area follows the diffusion equation approach, in which the orientation averages are computed as moments of the ODF. The ODF is the solution of the diffusion equation, and is usually approximated by some numerical scheme appropriate to the range of parameters of interest. Instead of solving the diffusion equation, Kumar and Ramamohan ${ }^{18}$ obtained the moments by directly integrating a set of equations governing the orientations of individual particles and employing a bruteforce computation. These equations were deterministic ordinary differential equations since Brownian effects were neglected in their work. A direct generalization of their method to systems with noise would involve numerically integrating a set of stochastic differential equations (Langevin equations) governing the evolution of the orientation vectors, but this is usually difficult and prone to error. In this work, therefore, we use a generalized Langevin equation method to evaluate the orientation moments, that leads to deterministic differential equations for the moments even for systems with Gaussian white noise. The basic ideas of this approach to suspension problems have been developed by Asokan et al. ${ }^{23}$ in an earlier work for the case of force-free particles and is easy to adapt to the present system involving dipolar particles exposed to an external field. A brief account of this procedure is as follows. We set up the Langevin equations governing the orientations of the particles by modifying the corresponding equations for force-free particles derived in Asokan et al. $^{23}$ By using suitable time-averaged forms of these equations we can obtain the exact equation of motion for any desired orientation moment. These equations for moments are deterministic, unlike the original Langevin equations that are stochastic in nature, but may not be closed and hence may not be easy to solve in general. However, by considering them in suitable pairs we can generate the moments by applying a brute-force computation on an ensemble of these equations. In the case of constant external force fields our results are in good agreement with other results in the literature obtained by the diffusion equation approach. For periodic forcing we demonstrate the possibility of chaotic behavior of the rheological properties that may not possibly be picked up by the diffusion equation approach. The chaotic response has been observed in some parameter regimes where Brownian diffusion is weak, a region where the problem becomes singular in the limit of zero diffusion. This is a significant advantage of the Langevin method when used along with the paired moment scheme presented in this work.

The rest of the paper is organized as follows. The Langevin equations for the orientation of the particles are obtained in Sec. II using results derived in Asokan et al. ${ }^{23}$ The expressions for the various stress components, including contributions from hydrodynamic forces, Brownian diffusion, and a constant external force field, are presented in Sec. III. A brute-force technique to compute the various orientation moments in suitable pairs is also described here. The intrinsic viscosity of the system is evaluated for various values of the diffusion coefficient and different strengths and orientations of the external field and compared with known results. In Sec. IV a periodic external force is considered for the case of fibers and the observed fluctuations in the apparent viscosity of the system are analyzed using dynamical and topological tools for various strengths of the shear and external fields. Finally, concluding remarks are given in Sec. V.

\section{THE THEORY}

We consider a dilute suspension of identical rigid, neutrally buoyant spheroids in an infinite incompressible Newtonian fluid subjected to a simple shear flow defined by a flow field, $\mathbf{v}=\dot{\gamma} y \mathbf{i}$, where $\dot{\gamma}$ is the shear rate, $y$ is the $y$ coordinate, and $\mathbf{i}$ is the unit vector in the $X$ direction. The volume concentration $\Phi$ of the particles in the solvent is assumed sufficiently small that hydrodynamic interactions among particles can be neglected. The shape of the spheroid varies depending on its aspect ratio defined by $r=a / b$, where $a$ and $b$ are, respectively, the polar and equatorial radii; spheres have $r=1$, and slender rods (fibers) correspond to $r \rightarrow \infty$. We choose a coordinate system that moves along with the particle and is affixed to its center of mass, thus neglecting any translatory motion, since it does not contribute to the bulk suspension properties in a dilute system. The orientation of the spheroid is then represented by a unit vector $\mathbf{u}$ placed along the major axis of the spheroid and the direction of $\mathbf{u}$ can be specified by the polar coordinates $(\theta, \phi)$, $\theta(0 \leqslant \theta \leqslant \pi)$ and $\phi(0 \leqslant \phi \leqslant 2 \pi)$ being the polar and azimuthal 
angles determined by the vector $\mathbf{u}$. An external force field may affect the local dynamics if the particles are dipolar and this may arise from several sources; ${ }^{4}$ (1) the particles have magnetic charges (e.g., ferromagnetic fibers in a ferrofluid) and the suspension is exposed to a magnetic field or (2) the particles are charged fibers (e.g., charged polymers in an aqueous solution) and may respond to an external electric field; or (3) they may possess a gravitational dipole and may be influenced by gravity (e.g., bioconvection set up by the swimming of certain microorganisms ${ }^{6,7}$ ). In all the above cases the external field can be modeled as the cross-product of the particle dipole moment $\mathbf{m}$ and the uniform external field vector $\mathbf{H}$;

$$
T^{\mathrm{ext}}=\mathbf{m} \times \mathbf{H} .
$$

The dipole moment $\mathbf{m}$ is assumed to be parallel to the particle symmetry axis, so that we can write

$$
\mathbf{m}=m \mathbf{u},
$$

where $m$ is the magnitude of the dipole moment. The time rate of change of the orientation vector $\mathbf{u}$ can be expressed as $\dot{\mathbf{u}}=\boldsymbol{\omega} \times \mathbf{u}$, where $\boldsymbol{\omega}$ is the angular velocity of the particle, an expression for which may be obtained by the angular momentum balance equation thus ${ }^{4}$ is

$$
\boldsymbol{\omega}=\boldsymbol{\Omega}+C[\mathbf{u} \times(\mathbf{E} \cdot \mathbf{u})]+\zeta_{\perp}^{-1} \mathbf{m} \times \mathbf{H} .
$$

Above, $C=\left(r^{2}-1\right) /\left(r^{2}+1\right)$ is a shape factor for the spheroid that takes the value -1 for disks, 0 for spheres, and +1 for long fibers, and $\mathbf{E}$ and $\boldsymbol{\Omega}$ are, respectively, the rate of deformation tensor and the vorticity vector for the flow, given by

$$
\begin{aligned}
& \mathbf{E}=\frac{1}{2}\left(\boldsymbol{\nabla} \mathbf{v}+\boldsymbol{\nabla} \mathbf{v}^{T}\right), \\
& \mathbf{\Omega}=\frac{1}{2}(\boldsymbol{\nabla} \times \mathbf{v}) .
\end{aligned}
$$

The effect of Brownian rotation can now be included by superposition, assuming that it causes the angular velocity to change by a white noise vector term; ${ }^{23}$

$$
\boldsymbol{\omega}=\boldsymbol{\Omega}+C[\mathbf{u} \times(\mathbf{E} \cdot \mathbf{u})]+\zeta_{\perp}^{-1} \mathbf{m} \times \mathbf{H}+\boldsymbol{\Gamma}(t) .
$$

The Cartesian components $\Gamma_{i}(t)$ are Gaussian random variables with zero mean and autocorrelation functions proportional to the $\delta$ function:

$$
\overline{\Gamma_{j}(t)}=0, \quad \overline{\Gamma_{i}(t) \Gamma_{j}\left(t^{\prime}\right)}=2 D \delta_{i j} \delta\left(t-t^{\prime}\right),
$$

where $\delta_{i j}$ is the Kronecker delta, $\delta(t)$ is the Dirac delta function, and $D$ is the spectral density, which in this case is equal to the rotary diffusivity defined by $D_{r}=k_{\mathrm{B}} T / \zeta_{\perp}$, where $\zeta_{\perp}$ represents the rotational resistance in the direction perpendicular to the particle symmetry axis, $k_{\mathrm{B}}$ is the Boltzmann constant, and $T$ is the absolute temperature. The overbars denote statistical averages over a large number of random variables. This leads to

$$
\dot{\mathbf{u}}=\boldsymbol{\Omega} \times \mathbf{u}+C[\mathbf{u} \times(\mathbf{E} \cdot \mathbf{u})] \times \mathbf{u}+\zeta_{\perp}^{-1} \mathbf{m} \times \mathbf{H} \times \mathbf{u}+\boldsymbol{\Gamma} \times \mathbf{u} .
$$

Equation (2) is the full Langevin equation for the Brownian spheroid in the presence of an external force field. The par- ticular form of the noise term in the above expression is derived in Asokan et al. ${ }^{23}$ for force-free particles by utilizing a generalized Langevin equation approach presented by Coffey et ll $^{24}$ The reader is referred to Asokan et al. ${ }^{23}$ for further details. In Cartesian components $u_{i}$ of $\mathbf{u}$ and $h_{i}$ of $\mathbf{H}, \mathrm{Eq}$. (2) can be written as

$$
\begin{aligned}
\dot{u}_{1}= & \dot{\gamma} C u_{2}\left(1-u_{1}^{2}\right)+\dot{\gamma}\left(\frac{1-C}{2}\right) u_{2}+\frac{m}{\zeta_{\perp}}\left[u_{3}\left(u_{3} h_{1}-u_{1} h_{3}\right)\right. \\
& \left.-u_{2}\left(u_{1} h_{2}-u_{2} h_{1}\right)\right]+\Gamma_{2}(t) u_{3}-\Gamma_{3}(t) u_{2}, \\
\dot{u}_{2}= & -\dot{\gamma} C u_{1} u_{2}^{2}-\dot{\gamma}\left(\frac{1-C}{2}\right) u_{1}+\frac{m}{\zeta_{\perp}}\left[u_{1}\left(u_{1} h_{2}-u_{2} h_{1}\right)\right. \\
& \left.-u_{3}\left(u_{2} h_{3}-u_{3} h_{2}\right)\right]+\Gamma_{3}(t) u_{1}-\Gamma_{1}(t) u_{3}, \\
\dot{u}_{3}= & -\dot{\gamma} C u_{1} u_{2} u_{3}+\frac{m}{\zeta_{\perp}}\left[u_{2}\left(u_{2} h_{3}-u_{3} h_{2}\right)-u_{1}\left(u_{3} h_{1}\right.\right. \\
& \left.\left.-u_{1} h_{3}\right)\right]-\Gamma_{1}(t) u_{2}-\Gamma_{2}(t) u_{1},
\end{aligned}
$$

where the $h_{i}$ denote the components of $\mathbf{H}$.

\section{THE STRESS TENSOR}

Strand and $\mathrm{Kim}^{4}$ obtained the following expression for the mean dimensional stress in the suspension that includes contributions from both rotary Brownian diffusion and an external force field,

$$
\begin{aligned}
\boldsymbol{\sigma}= & -p \boldsymbol{\delta}+2 \eta_{s} \mathbf{E}+2 \eta_{s} \Phi\left\{2 A_{H} \mathbf{E}:\langle\mathbf{u u u u}\rangle\right. \\
& +2 B_{H}\left(\mathbf{E} \cdot\langle\mathbf{u u}\rangle+\langle\mathbf{u u}\rangle \cdot \mathbf{E}-\frac{2}{3} \boldsymbol{\delta} \mathbf{E}:\langle\mathbf{u u}\rangle\right)+C_{H} \mathbf{E} \\
& +F_{H} D_{r}\left(\langle\mathbf{u u}\rangle-\frac{1}{3} \boldsymbol{\delta}\right)+3 D_{0} \frac{m}{k T}\left(\frac{(1-C)}{2}\left\langle\mathbf{u} \mathbf{H}_{\perp}\right\rangle\right. \\
& \left.\left.-\frac{(1+C)}{2}\left\langle\mathbf{H}_{\perp} \mathbf{u}\right\rangle\right)\right\} .
\end{aligned}
$$

The angular brackets indicate orientation averages over an ensemble of particles. Here $p$ is the pressure, $\eta_{s}$ is the viscosity of the solvent, $D_{0}$ is the rotary diffusivity of a sphere of volume equal to that of the particle, and $\mathbf{H}_{\perp}=\mathbf{H} \cdot(\boldsymbol{\delta}$ $-\mathbf{u u})$ is the component of the external force $\mathbf{H}$ in the direction perpendicular to the particle symmetry axis. The other coefficients $A_{H}, B_{H}, C_{H}, F_{H}$, called the stress coefficients, are functions of the shape of the particle, the general expressions for which are given in Strand and Kim. ${ }^{4}$ For general spheroids these coefficients involve certain elliptic integrals, but for some limiting cases such as spheres and long fibers, these expressions can be considerably simplified. The limiting values of the coefficients for long fibers $(r \rightarrow \infty)$ and near spheres $(r \rightarrow 1)$ are listed in Table I. ${ }^{4}$

The rheological properties of a suspension are usually expressed in terms of material functions, such as the apparent viscosities and the first and second normal stress differences. For dilute suspensions these quantities involve the limit $\Phi \rightarrow 0$ and are therefore called intrinsic properties. The ex- 
TABLE I. The limiting values of the stress coefficients.

\begin{tabular}{ccc}
\hline \hline Coefficient & $r \rightarrow \infty$ & $r=1+\epsilon(\epsilon \rightarrow 0)$ \\
\hline$A_{H}$ & $\frac{r^{2}}{4[\ln (2 r)-3 / 2]}$ & $\frac{395}{294} \epsilon^{2}$ \\
$B_{H}$ & $\frac{3 \ln (2 r)-11 / 2}{r^{2}}$ & $\frac{15}{28} \epsilon-\frac{895}{1175} \epsilon^{2}$ \\
$C_{H}$ & 2 & $\frac{5}{2}-\frac{5}{7} \epsilon+\frac{235}{294} \epsilon^{2}$ \\
$F_{H}$ & $\frac{3 r^{2}}{\ln (2 r)-1 / 2}$ & $9 \epsilon$ \\
\hline \hline
\end{tabular}

pressions for these rheological properties for the case of simple shear, defined below, can be obtained from Eq. (4):

$$
\begin{aligned}
& {\left[\eta_{1}\right]=\lim _{\Phi \rightarrow 0}\left(\frac{\sigma_{x y}-\eta_{s} \dot{\gamma}}{\Phi \eta_{s} \dot{\gamma}}\right)} \\
& =4 A_{H}\left\langle u_{1}^{2} u_{2}^{2}\right\rangle+2 B_{H}\left\langle u_{1}^{2}+u_{2}^{2}\right\rangle+C_{H}+\left(\frac{2 F_{H}}{\mathrm{Pe}}\right)\left\langle u_{1} u_{1}\right\rangle \\
& +\left(\frac{2 F_{H}}{3 C P e}\right)\left[C\left\langle\left(\Sigma k_{i} u_{i}\right) u_{1} u_{2}\right\rangle+\left(\frac{1-C}{2}\right)\left\langle u_{1}\right\rangle k_{2}\right. \\
& \left.-\left(\frac{1+C}{2}\right)\left\langle u_{2}\right\rangle k_{1}\right] \\
& {\left[\eta_{2}\right]=\lim _{\Phi \rightarrow 0}\left(\frac{\sigma_{y x}-\eta_{s} \dot{\gamma}}{\Phi \eta_{s} \dot{\gamma}}\right)} \\
& =4 A_{H}\left\langle u_{1}^{2} u_{2}^{2}\right\rangle+2 B_{H}\left\langle u_{1}^{2}+u_{2}^{2}\right\rangle+C_{H}+\left(\frac{2 F_{H}}{\mathrm{Pe}}\right)\left\langle u_{1} u_{1}\right\rangle \\
& +\left(\frac{2 F_{H}}{3 C P e}\right)\left[C\left\langle\left(\Sigma k_{i} u_{i}\right) u_{1} u_{2}\right\rangle+\left(\frac{1-C}{2}\right)\left\langle u_{2}\right\rangle k_{1}\right. \\
& \left.-\left(\frac{1+C}{2}\right)\left\langle u_{1}\right\rangle k_{2}\right] \text {, } \\
& {\left[\tau_{1}\right]=\lim _{\Phi \rightarrow 0}\left(\frac{\sigma_{x x}-\sigma_{z z}}{\Phi \eta_{s} \dot{\gamma}}\right)} \\
& =4 A_{H}\left(\left\langle u_{1}^{3} u_{2}\right\rangle-\left\langle u_{1} u_{2} u_{3}^{2}\right\rangle\right)+4 B_{H}\left\langle u_{1} u_{2}\right\rangle+\left(\frac{2 F_{H}}{\mathrm{Pe}}\right) \\
& \times\left\langle u_{1}^{2}-u_{3}^{2}\right\rangle+\left(\frac{2 F_{H}}{3 P e}\right)\left[\left\langle\left(\Sigma k_{i} u_{i}\right) u_{1}^{2}\right\rangle-\left\langle\left(\Sigma k_{i} u_{i}\right) u_{3}^{2}\right\rangle\right. \\
& \left.+k_{3}\left\langle u_{3}\right\rangle-k_{1}\left\langle u_{1}\right\rangle\right] \text {, } \\
& {\left[\tau_{2}\right]=\lim _{\Phi \rightarrow 0}\left(\frac{\sigma_{y y}-\sigma_{z z}}{\Phi \eta_{s} \dot{\gamma}}\right)} \\
& =4 A_{H}\left(\left\langle u_{1} u_{2}^{3}\right\rangle-\left\langle u_{1} u_{2} u_{3}^{2}\right\rangle\right)+4 B_{H}\left\langle u_{1} u_{2}\right\rangle+\left(\frac{2 F_{H}}{\mathrm{Pe}}\right) \\
& \times\left\langle u_{2}^{2}-u_{3}^{2}\right\rangle+\left(\frac{2 F_{H}}{3 \mathrm{Pe}}\right)\left[\left\langle\left(\Sigma k_{i} u_{i}\right) u_{2}^{2}\right\rangle-\left\langle\left(\Sigma k_{i} u_{i}\right) u_{3}^{2}\right\rangle\right. \\
& \left.+k_{3}\left\langle u_{3}\right\rangle-k_{2}\left\langle u_{2}\right\rangle\right] \text {. }
\end{aligned}
$$

For compactness we have used the Cartesian components to denote the various averages. In obtaining the above forms for the intrinsic properties from Eq. (4) we have followed the scaling of Strand and $\mathrm{Kim}^{4}$ to make comparisons easier, namely, time is scaled with respect to $6 D_{r}$ and force with respect to $k_{\mathrm{B}} T$. The dimensionless quantity $\mathrm{Pe}=\dot{\gamma} / D_{r}$, called the Péclet number, measures the relative strengths of the fluxes due to shear and diffusion. The external force is assumed constant (i.e., without fluctuations), the scaled dimensionless form of which is denoted by $\mathbf{k}$ in the above equations with $\mathbf{k}=m \mathbf{H} / k_{\mathrm{B}} T$. We have also used the fact that $D_{0} / D_{r}=F_{H} /(9 C)$ to clear the expressions of the constant $D_{0}$. In the scaled form the Langevin equations for the orientation behavior of the spheroids, Eq. (3), after being converted to spherical coordinates, take the following form:

$$
\begin{aligned}
& \dot{\theta}=h_{1}-\sin \phi \Gamma_{1}(t)+\cos \phi \Gamma_{2}(t), \\
& \dot{\phi}=h_{2}-\cot \theta \cos \phi \Gamma_{1}(t)-\sin \phi \cot \theta \Gamma_{2}(t)+\Gamma_{3}(t),
\end{aligned}
$$

where $h_{1}$ and $h_{2}$ are the deterministic parts of the equation, given by

$$
\begin{aligned}
h_{1}= & \left(\frac{\mathrm{Pe}}{6}\right) C \sin \theta \cos \theta \sin \phi \cos \phi \\
& +\frac{1}{6}\left(k_{1} \cos \theta \cos \phi+k_{2} \cos \theta \sin \phi-k_{3} \sin \theta\right), \\
h_{2}= & -\left(\frac{\mathrm{Pe}}{6}\right) C \sin ^{2} \phi-\left(\frac{\mathrm{Pe}}{6}\right)\left(\frac{1-C}{2}\right) \\
& +\frac{1}{6}\left(-k_{1} \sin \phi+k_{2} \cos \phi\right)\left(\frac{1}{\sin \theta}\right) .
\end{aligned}
$$

Note that the Gaussian random variables $\Gamma_{i}(t)$ now satisfy, after scaling, Eq. (1) with $D=1 / 6$;

$$
\overline{\Gamma_{j}(t)}=0, \quad \overline{\Gamma_{i}(t) \Gamma_{j}\left(t^{\prime}\right)}=\frac{1}{3} \delta_{i j} \delta\left(t-t^{\prime}\right) .
$$

We now evaluate the intrinsic viscosity $[\eta]=\left[\eta_{1}\right]$ for a number of ranges of $\mathbf{k}$ and $\mathrm{Pe}$ and compare the results with those of Strand and $\mathrm{Kim}^{4}$ and Brenner and Weissman. ${ }^{10}$

To compute the various orientation moments appearing in the above expressions for rheological parameters, we revert to the techniques developed in Asokan et al. ${ }^{23}$ Essentially this procedure simplifies the computation of ensemble averages in nonlinear systems with noise governed by stochastic differential equations by simulating a set of ordinary differential equations obtained by suitable time averaging. Note that Eq. (5) is a pair of nonlinear Langevin equations with multiplicative Gaussian noise terms describing the dynamics of the vector $\boldsymbol{\theta}=(\theta, \phi)$. Denoting the noise coefficients by $g_{i j}$, it can be written concisely as

$$
\dot{\theta}_{i}(t)=h_{i}[\boldsymbol{\theta}(t), t]+g_{i j}[\boldsymbol{\theta}(t), t] \Gamma_{j}(t),
$$

where $\theta_{1}=\theta, \theta_{2}=\phi$. Using these equations and following the procedure outlined in Asokan et al., ${ }^{23}$ one obtains the following equation for the dynamics of any moment $\langle B(\theta, \phi)\rangle$ : 


$$
\begin{aligned}
\frac{\mathrm{d}}{\mathrm{d} t}\langle B(\theta, \phi)\rangle= & \left\langle f_{1} h_{1}\right\rangle+\left\langle f_{2} h_{2}\right\rangle \\
& +\frac{1}{6}\left\langle g_{k j} \frac{\partial}{\partial \theta_{k}}\left(f_{1} g_{1 j}+f_{2} g_{2 j}\right)\right\rangle,
\end{aligned}
$$

where we have used Einstein's summation convention in the last term. The ensemble average in the above is taken over a set of tracer orientations $(\theta, \phi)$ in orientation space. ${ }^{23}$ For a given moment, however, Eq. (8) may not, in general, be closed and easy to solve, hence we use an alternate method to generate the desired moments in suitable pairs. Note that the moment equations, Eq. (8), corresponding to any two moments, $\left\langle B_{1}(\theta, \phi)\right\rangle$ and $\left\langle B_{2}(\theta, \phi)\right\rangle$, are collectively equivalent to an ensemble of the following simultaneous equations over a set of tracer orientations:

$$
\begin{aligned}
\dot{\theta}= & h_{1}+\frac{1}{6 \Delta}\left(f_{2}^{\prime} g_{k j} \frac{\partial}{\partial \theta_{k}}\left(f_{1} g_{1 j}+f_{2} g_{2 j}\right)\right. \\
& \left.-f_{2} g_{k j} \frac{\partial}{\partial \theta_{k}}\left(f_{1}^{\prime} g_{1 j}+f_{2}^{\prime} g_{2 j}\right)\right), \\
\dot{\phi}= & h_{2}-\frac{1}{6 \Delta}\left(f_{1}^{\prime} g_{k j} \frac{\partial}{\partial \theta_{k}}\left(f_{1} g_{1 j}+f_{2} g_{2 j}\right)\right. \\
& \left.-f_{1} g_{k j} \frac{\partial}{\partial \theta_{k}}\left(f_{1}^{\prime} g_{1 j}+f_{2}^{\prime} g_{2 j}\right)\right) .
\end{aligned}
$$

In the above, $f_{i}$ are the partial derivatives of $B_{1}$ and $f_{i}^{\prime}$ are those of $B_{2}$ and the pair $B_{1}, B_{2}$ may be so chosen that $\Delta$ $=f_{1} f_{2}^{\prime}-f_{1}^{\prime} f_{2}$ is not identically zero. The moments are now obtained by employing a brute-force computation on an ensemble of equations [Eq. (9)] over a set of initial conditions in the orientation space. Fix a positive integer $n$ and define $n^{2}$ points on the $\theta \phi$ space $[0, \pi] \times[0,2 \pi]$, given by

$$
\left.\begin{array}{rl}
\theta_{i 0} & =\left(\theta_{i}+\theta_{i+1}\right) / 2 \\
\phi_{j 0} & =\left(\theta_{j}+\theta_{j+1}\right) / 2
\end{array}\right\} i, j=0,1, \ldots, n,
$$

where $\theta_{i}=\cos ^{-1}[(2 i / n)-1]$ and $\phi_{i}=2 \pi / n, i=0,1 \cdots n$. This corresponds to a set of initial conditions distributed nearly uniformly over the orientation space. We then consider $n^{2}$ copies of Eqs. (9) over the above set of initial conditions and numerically integrate each pair simultaneously using the integrator odeint of Press et al. ${ }^{25}$ with adaptive step-size control within a tolerance of $0.001 \%$. From the values $\left(\theta_{i t}, \phi_{j t}\right)$ at the end of each iterative step, the numbers $B_{1}\left(\theta_{i t}, \phi_{j t}\right)$ and $B_{2}\left(\theta_{i t}, \phi_{j t}\right)$ are computed and averaged to get an estimate of the moment, and thus ${ }^{23}$

$$
\begin{aligned}
\left\langle B_{i}(\theta, \phi)\right\rangle & =\frac{\iint B_{i}(\theta, \phi) \delta\left(\theta-\theta_{i t}\right) \delta\left(\phi-\phi_{j t}\right) \mathrm{d}(\cos \theta) \mathrm{d} \phi}{\iint \delta\left(\theta-\theta_{i t}\right) \delta\left(\phi-\phi_{j t}\right) \mathrm{d}(\cos \theta) \mathrm{d} \phi} \\
& =\frac{1}{n^{2}} \sum_{i=1}^{n} \sum_{j=1}^{n} B_{i}\left(\theta_{i t}, \phi_{j t}\right) .
\end{aligned}
$$

\begin{tabular}{|c|c|}
\hline Moments & Noise terms \\
\hline$\left\{\begin{array}{l}\left\langle u_{1}^{2} u_{2}^{2}\right\rangle \\
\left\langle u_{3}^{2}\right\rangle\end{array}\right.$ & $\left\{\begin{array}{l}2 \sin ^{2} \theta-20 \sin ^{4} \theta \sin ^{2} \phi \cos ^{2} \phi \\
2\left(3 \cos ^{2} \theta-1\right)\end{array}\right.$ \\
\hline$\left\{\begin{array}{l}\left\langle u_{1}^{2}\right\rangle \\
\left\langle u_{2}^{2}\right\rangle\end{array}\right.$ & $\left\{\begin{array}{l}2-6 \sin ^{2} \theta \cos ^{2} \phi \\
2-6 \sin ^{2} \theta \cos ^{2} \phi\end{array}\right.$ \\
\hline$\left\{\begin{array}{l}\left\langle u_{1}^{2} u_{2}\right\rangle \\
\left\langle u_{1} u_{2}^{2}\right\rangle\end{array}\right.$ & $\left\{\begin{array}{l}2 \sin \theta \sin \phi-12 \sin ^{3} \theta \sin \phi \cos ^{2} \phi \\
2 \sin \theta \cos \phi-12 \sin ^{3} \theta \cos \phi \sin ^{2} \phi\end{array}\right.$ \\
\hline$\left\{\begin{array}{l}\left\langle u_{1} u_{2} u_{3}\right\rangle \\
\left\langle u_{3}^{3}\right\rangle\end{array}\right.$ & $\left\{\begin{array}{c}-6 \cos \theta \sin ^{2} \theta \sin 2 \phi \\
-3(\cos \theta+\cos 3 \theta)\end{array}\right.$ \\
\hline$\left\{\begin{array}{l}\left\langle u_{1}\right\rangle \\
\left\langle u_{2}\right\rangle\end{array}\right.$ & $\left\{\begin{array}{l}-2 \sin \theta \cos \phi \\
-2 \sin \theta \sin \phi\end{array}\right.$ \\
\hline$\left\{\begin{array}{l}\left\langle u_{1} u_{2}\right\rangle \\
\left\langle u_{3}^{2}\right\rangle\end{array}\right.$ & $\left\{\begin{array}{l}-3 \sin ^{2} \theta \sin 2 \phi \\
-2\left(3 \cos ^{2} \theta-1\right)\end{array}\right.$ \\
\hline$\left\{\begin{array}{l}\left\langle u_{1} u_{2}^{3}\right\rangle \\
\left\langle u_{1}^{3} u_{2}\right\rangle\end{array}\right.$ & $\left\{\begin{array}{l}3 \sin ^{2} \theta \sin 2 \phi-10 \sin ^{4} \theta \sin ^{2} \phi \sin 2 \phi \\
3 \sin ^{2} \theta \sin 2 \phi-10 \sin ^{4} \theta \cos ^{2} \phi \sin 2 \phi\end{array}\right.$ \\
\hline$\left\{\begin{array}{l}\left\langle u_{1}^{2} u_{3}\right\rangle \\
\left\langle u_{3} u_{2}^{2}\right\rangle\end{array}\right.$ & $\left\{\begin{array}{l}2 \cos \theta-12 \sin ^{2} \theta \cos ^{2} \phi \cos \theta \\
2 \cos \theta-12 \sin ^{2} \theta \sin ^{2} \phi \cos \theta\end{array}\right.$ \\
\hline$\left\{\begin{array}{l}\left\langle u_{1}^{3}\right\rangle \\
\left\langle u_{2}^{3}\right\rangle\end{array}\right.$ & $\left\{\begin{array}{l}6 \sin \theta \cos \phi-12 \sin ^{3} \theta \cos ^{3} \phi \\
6 \sin \theta \sin \phi-12 \sin ^{3} \theta \sin ^{3} \phi\end{array}\right.$ \\
\hline
\end{tabular}

This is repeated for successive time steps using, at each step, the values $\left(\theta_{i}, \phi_{j}\right)$ obtained from numerical integration, and continued until the values of the moments stabilize. This procedure generates a numerical approximation to the dynamics of the moments $B_{1}$ and $B_{2}$.
TABLE II. The various moments in the expressions for the rheological parameters and the corresponding noise terms.

Table II lists some of the moments appearing in the expressions for the rheological parameters and their pairing used in our simulations, along with the noise term [the last term on the right of Eq. (8)] corresponding to each moment. Others can be easily obtained as linear combinations of those in the table. The choice of the moment pairs was made based on the symmetry in the noise terms (Table II) and the condition that $\Delta$ be not identically zero for the chosen pairs. As in Asokan et al. ${ }^{23}$ the number of initial conditions was kept at $n^{2}=100$ at which the results more or less stabilized.

Figure 1 shows plots of the intrinsic viscosity $\left[\eta_{1}\right]$ versus the external field strength $\mathbf{k}$ for a fixed shear rate $P_{e}$ $=2$, and two different external field orientations. The azimuthal direction $\phi$ is held at $90^{\circ}$ while the polar directions are $45^{\circ}$ and $90^{\circ}$. The results for three different aspect ratios are shown: prolate spheroids with $r=1.6$; oblate spheroids with $r=0.4$ and spheres. The calculations are greatly simplified for spheres for which $A_{H}=B_{H}=F_{H}=0, C_{H}=5 / 2$ and $F_{H} / P_{e}=9 / 2$ (see Table I). These results are in good agreement with those of Strand and $\mathrm{Kim}^{4}$ and Brenner and Weissman ${ }^{10}$ (see Fig. 2 in Strand and $\mathrm{Kim}^{4}$ ). For external force fields higher $(>5),\left[\eta_{1}\right]$ stabilizes rapidly to the values shown in the figure, but for smaller field strengths it takes longer for the values to stabilize.

The effect of the Brownian and shear parameters on the intrinsic viscosity $\left[\eta_{1}\right]$ are plotted in Fig. 2 for spheroids of aspect ratio $r=0.4,1.0$, and 1.6 at two different field orientations, keeping the strength of the external field constant at $\mathbf{k}=1$. A comparison with Fig. 3 in Strand and $\mathrm{Kim}^{4}$ shows 


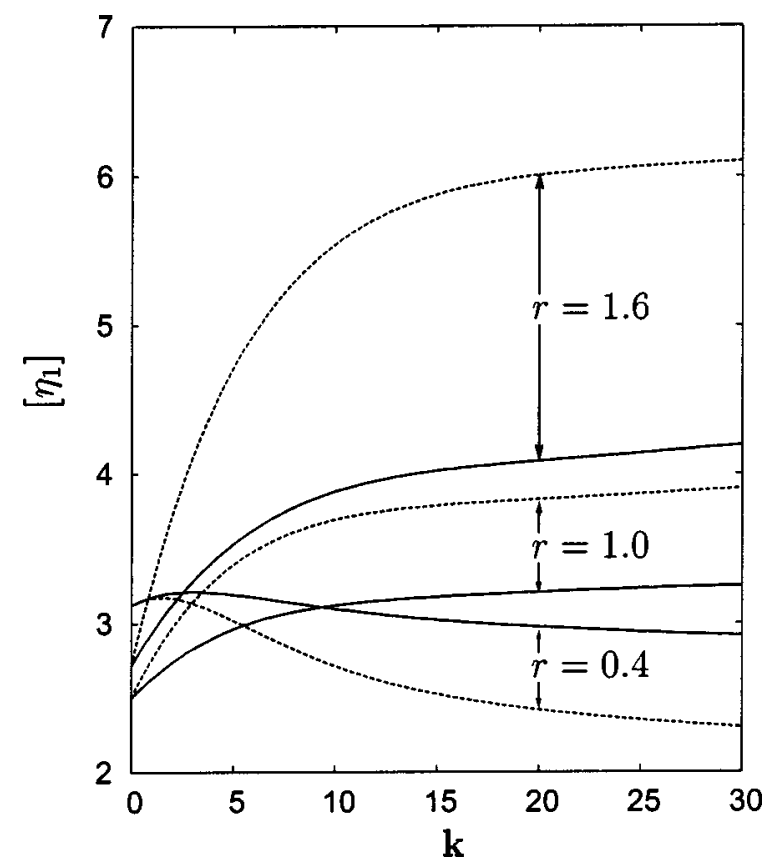

FIG. 1. Plot of intrinsic viscosity $\left[\eta_{1}\right]$ for dipolar suspensions for various strengths of the external field, for spheroids of aspect ratio $r=0.4,1.0$, and 1.6. Results for $\mathbf{k} \|(0,1,1)$ (solid lines) and $\mathbf{k} \|(0,1,0)$ (dotted lines) are shown. Compare with Fig. 2 of Strand and Kim (Ref. 4).

that the results are in good agreement with those obtained by the diffusion equation method.

\section{PERIODIC FORCING}

If the external force is periodic, the dynamics and the rheology of the suspension considered here can in some

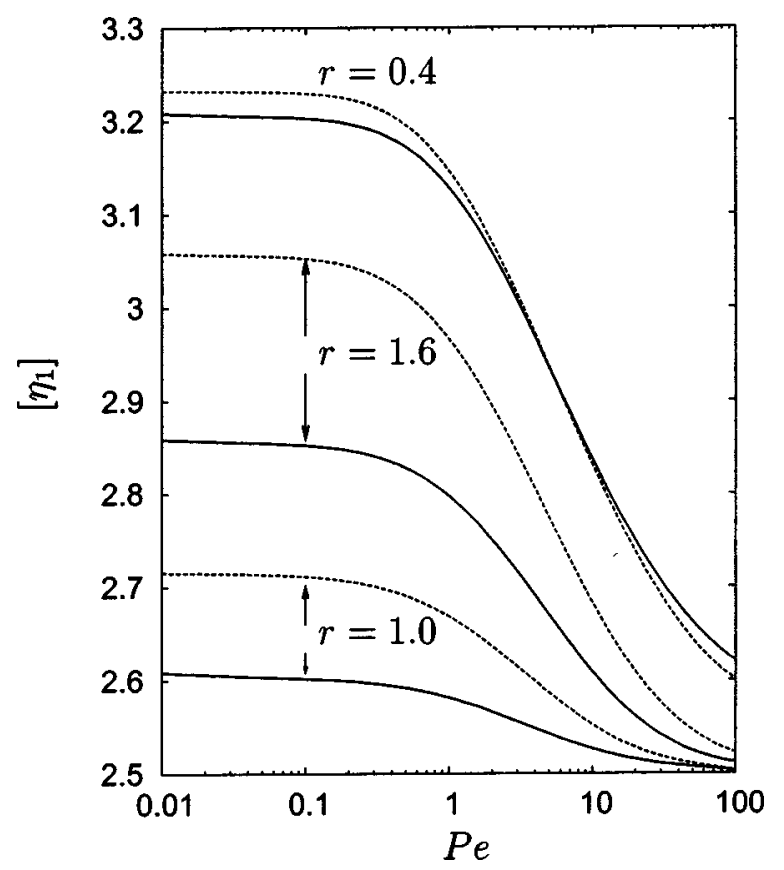

FIG. 2. Plot of intrinsic viscosity $\left[\eta_{1}\right]$ for dipolar suspensions for various values of Pe, for spheroids of aspect ratio $r=0.4,1.0$, and 1.6. Results for $\mathbf{k} \|(0,1,1)$ (solid lines) and $\mathbf{k} \|(0,1,0)$ (dotted lines) are shown. Compare with Fig. 3 of Strand and Kim (Ref. 4). cases exhibit complex behavior. The experimental feasibility of setting up a suspension system under constant external forcing has been reported by many authors ${ }^{26-29}$ and periodic forcing should cause no additional problem. Most of the work on suspensions under periodic external forcing has been done on electrorheological and magnetorheological fluids. Ramamohan et al. have studied extensively suspensions of periodically driven dipolar spheroids in the limit of negligible or zero Brownian motion and demonstrated the existence of parametric regimes where both the rotational dynamics and the intrinsic suspension properties evolve chaotically. ${ }^{16-19}$ A new type of class-I intermittency route to chaos has also been shown to exist in the system they studied, ${ }^{20}$ thus providing an example of a physically realizable system showing a nonhysteretic form of class-I intermittency. Further, a new and easy to implement chaos control algorithm has been developed, which leads to a very efficient scheme for separating particles. ${ }^{21}$ The microparticle suspension they studied is also one of the few examples of a physically realizable system showing spatiotemporal chaos and nontrivial collective behavior. ${ }^{22}$ This system thus represents one of the simplest physically realizable systems for studying the average behavior of a large number of individually chaotically varying elements. The system considered here is therefore important, both from a theoretical as well a practical point of view.

All the above investigations were limited to zero or negligible Brownian motion. The method outlined in the previous section can be used to generalize these studies by including the additional contributions to the stress due to Brownian rotation of the individual particles. Under a sinusoidally varying force field the Langevin equation (2) modifies to

$$
\begin{aligned}
\dot{\mathbf{u}}= & \boldsymbol{\Omega} \times \mathbf{u}+C[\mathbf{u} \times(E \cdot \mathbf{u})] \times \mathbf{u}+\zeta_{\perp}^{-1} \mathbf{m} \times \mathbf{H} \cos (\omega t) \times \mathbf{u} \\
& +\boldsymbol{\Gamma} \times \mathbf{u},
\end{aligned}
$$

where $\omega$ is the frequency of the external driver. The analysis in this section will be restricted to the case of slender rods (fibers) that correspond to the limit $r \rightarrow \infty$, so that the stress coefficients take a particularly simple form. We choose a scaling that is appropriate to explore the system behavior vis a vis change in the Brownian flux, so time is scaled with respect to the shear rate $(\dot{\gamma})$ and force with $\dot{\gamma} \zeta_{\perp}$, and write $\mathbf{k}=\left(m \mathbf{H} / \dot{\gamma} \zeta_{\perp}\right) \cos (\omega t)$. In this setting, the Langevin equation (10) takes the following form in spherical coordinates:

$$
\begin{aligned}
& \dot{\theta}=h_{1}-\sin \phi \Gamma_{1}(t)+\cos \phi \Gamma_{2}(t), \\
& \dot{\phi}=h_{2}-\cot \theta \cos \phi \Gamma_{1}(t)-\sin \phi \cot \theta \Gamma_{2}(t)+\Gamma_{3}(t),
\end{aligned}
$$

where $h_{1}$ and $h_{2}$ are, as before, the deterministic parts given by

$$
\begin{aligned}
h_{1}= & C \sin \theta \cos \theta \sin \phi \cos \phi+\left(k_{1} \cos \theta \cos \phi\right. \\
& \left.+k_{2} \cos \theta \sin \phi-k_{3} \sin \theta\right), \\
h_{2}= & -C \sin ^{2} \phi-\left(\frac{1-C}{2}\right)+\left(-k_{1} \sin \phi+k_{2} \cos \phi\right) \\
& \times\left(\frac{1}{\sin \theta}\right) .
\end{aligned}
$$




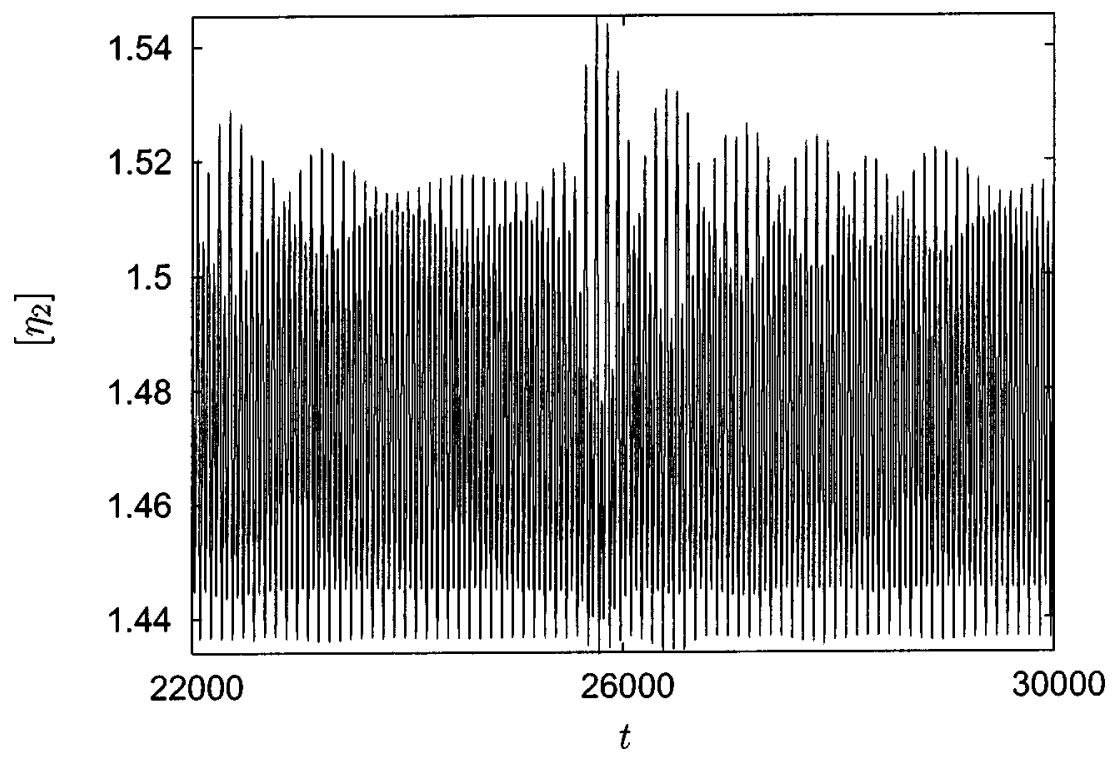

FIG. 3. A part of the time series of $\left[\eta_{2}\right]$ for $\widetilde{\mathrm{P}}=0.01, k_{1}=k_{3}=0, k_{2}$ $=0.1$, and $\omega=1$.
In the present scaling $\Gamma(t)$ satisfy (1) with $D=D_{r} / \dot{\gamma}$, which we denote by $\widetilde{P}$, so that $\widetilde{P} e=0$ when Brownian motion is switched off. The evolution equations (9) for the tracer orientations corresponding to the moments $\left\langle B_{1}\right\rangle$ and $\left\langle B_{2}\right\rangle$ now become

$$
\begin{aligned}
\dot{\theta}= & h_{1}+\frac{\widetilde{\mathrm{P}} \mathrm{e}}{\Delta}\left(f_{2}^{\prime} g_{k j} \frac{\partial}{\partial \theta_{k}}\left(f_{1} g_{1 j}+f_{2} g_{2 j}\right)\right. \\
& \left.-f_{2} g_{k j} \frac{\partial}{\partial \theta_{k}}\left(f_{1}^{\prime} g_{1 j}+f_{2}^{\prime} g_{2 j}\right)\right), \\
\dot{\phi}= & h_{2}-\frac{\widetilde{\mathrm{P}}}{\Delta}\left(f_{1}^{\prime} g_{k j} \frac{\partial}{\partial \theta_{k}}\left(f_{1} g_{1 j}+f_{2} g_{2 j}\right)\right. \\
& \left.-f_{1} g_{k j} \frac{\partial}{\partial \theta_{k}}\left(f_{1}^{\prime} g_{1 j}+f_{2}^{\prime} g_{2 j}\right)\right) .
\end{aligned}
$$

The limiting values of the stress coefficients for the case of fibers, given in Table I, can be used to further simplify the expressions for the rheological parameters by taking advantage of the following limits:

$$
\begin{array}{ll}
\lim _{r \rightarrow \infty} A_{H} B_{H}=\frac{3}{4}, & \lim _{r \rightarrow \infty} C_{H} B_{H}=0, \\
\lim _{r \rightarrow \infty} B_{H} B_{H}=0, & \lim _{r \rightarrow \infty} F_{H} B_{H}=9 .
\end{array}
$$

Hence, by scaling each of the rheological parameters with $B_{H}$ and taking a second limit as $r \rightarrow \infty$, we get

$$
\begin{aligned}
{\left[\eta_{1}\right]=} & \lim _{\substack{\Phi \rightarrow 0 \\
r \rightarrow \infty}}\left[\left(\frac{\sigma_{x y}-\eta_{s} \dot{\gamma}}{\Phi \eta_{s} \dot{\gamma}}\right) B_{H}\right] \\
= & 3\left\langle u_{1}^{2} u_{2}^{2}\right\rangle+18 \widetilde{\mathrm{P}}\left\langle u_{1} u_{1}\right\rangle+\left(\frac{6 \tilde{\mathrm{Pe}}}{C}\right) \\
& \times\left[C\left\langle\left(\sum k_{i} u_{i}\right) u_{1} u_{2}\right\rangle+\left(\frac{1-C}{2}\right)\left\langle u_{1}\right\rangle k_{2}\right. \\
& \left.-\left(\frac{1+C}{2}\right)\left\langle u_{2}\right\rangle k_{1}\right],
\end{aligned}
$$

$$
\begin{aligned}
& {\left[\eta_{2}\right]=\lim _{\substack{\Phi \rightarrow 0 \\
r \rightarrow \infty}}\left[\left(\frac{\sigma_{y x}-\eta_{s} \dot{\gamma}}{\Phi \eta_{s} \dot{\gamma}}\right) B_{H}\right]} \\
& =3\left\langle u_{1}^{2} u_{2}^{2}\right\rangle+18 \tilde{\mathrm{P}}\left\langle u_{1} u_{1}\right\rangle \\
& +\left(\frac{6 \tilde{\mathrm{P}} \mathrm{e}}{C}\right)\left[C\left\langle\left(\Sigma k_{i} u_{i}\right) u_{1} u_{2}\right\rangle+\left(\frac{1-C}{2}\right)\left\langle u_{2}\right\rangle k_{1}\right. \\
& \left.-\left(\frac{1+C}{2}\right)\left\langle u_{1}\right\rangle k_{2}\right] \text {, } \\
& {\left[\tau_{1}\right]=\lim _{\substack{\Phi \rightarrow 0 \\
r \rightarrow \infty}}\left[\left(\frac{\sigma_{x x}-\sigma_{z z}}{\Phi \eta_{s} \dot{\gamma}}\right) B_{H}\right]} \\
& =3\left(\left\langle u_{1}^{3} u_{2}\right\rangle-\left\langle u_{1} u_{2} u_{3}^{2}\right\rangle\right) \\
& +18 \widetilde{\operatorname{Pe}}\left\langle u_{1}^{2}-u_{3}^{2}\right\rangle+6 \widetilde{\operatorname{Pe}}\left[\left\langle\left(\Sigma k_{i} u_{i}\right) u_{1}^{2}\right\rangle\right. \\
& \left.-\left\langle\left(\sum k_{i} u_{i}\right) u_{3}^{2}\right\rangle+k_{3}\left\langle u_{3}\right\rangle-k_{1}\left\langle u_{1}\right\rangle\right] \text {, } \\
& {\left[\tau_{2}\right]=\lim _{\substack{\Phi \rightarrow 0 \\
r \rightarrow \infty}}\left[\left(\frac{\sigma_{y y}-\sigma_{z z}}{\Phi \eta_{s} \dot{\gamma}}\right) B_{H}\right]} \\
& =3\left(\left\langle u_{1} u_{2}^{3}\right\rangle-\left\langle u_{1} u_{2} u_{3}^{2}\right\rangle\right) \\
& +18 \widetilde{\operatorname{Pe}}\left\langle u_{2}^{2}-u_{3}^{2}\right\rangle+6 \widetilde{\operatorname{Pe}}\left[\left\langle\left(\Sigma k_{i} u_{i}\right) u_{2}^{2}\right\rangle\right. \\
& \left.-\left\langle\left(\Sigma k_{i} u_{i}\right) u_{3}^{2}\right\rangle+k_{3}\left\langle u_{3}\right\rangle-k_{2}\left\langle u_{2}\right\rangle\right] \text {. }
\end{aligned}
$$

\section{ANALYSIS OF THE TIME SERIES}

We generated a time series for each of the bulk suspension parameters above over a period of 100000 dimensionless time units using the computational techniques developed in the previous section and deleted the first 20000 data points to remove any transients. Figure 3 shows a part of the time series corresponding to the set of parameters $k_{1}=k_{3}$ $=0, k_{2}=0.10, \omega=1$, and $\widetilde{P}=0.01$ (weak diffusion), and it is clear from the figure that the apparent viscosity exhibits persistent temporal fluctuations. A detailed study of these fluc- 


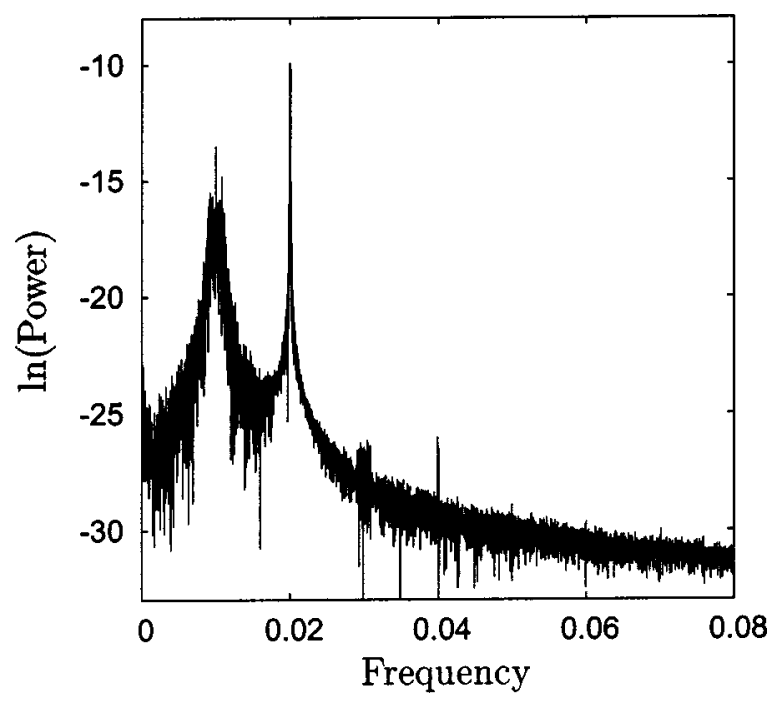

FIG. 4. Plot of the power versus frequency of $\left[\eta_{2}\right]$. The figure is typical of both chaotic and linear stochastic signals.

tuations using the tools of nonlinear time series analysis may reveal significant features of the dynamical system. A critical review of the various topological and geometrical methods for analyzing nonlinear data can be found in Kantz. ${ }^{30} \mathrm{We}$ used the softwares TISEAN ${ }^{31}$ and Chaos Data Analyzer Professional Version 2.1 of the Academic Software Library of the American Physical Society for performing the tests on the time series.

Figure 4 plots the frequency decomposition of the shear stress, which shows a broadband spectrum decaying exponentially with frequency. This is characteristic of both deterministic chaos and linear autocorrelated noise ${ }^{32,33}$ and suggests a further analysis of the system. The first step in any such investigation is the characterization of the attractor, a bounded subset of the phase space to which the system behavior eventually converges. This is usually done by reconstructing the attractor of the system from the time series using delay coordinates, a technique first suggested by Packard et $a l^{34}$ and successfully used by many others. The embedding theorems of Takens ${ }^{35}$ and its extensions ${ }^{36,37}$ elucidate the mathematical theory behind delay reconstruction. Roughly, the embedding theorems assert that for deterministic systems, the dynamics of the $n$-dimensional state vector $\mathbf{x}(t)$ can be recaptured from the dynamics of the delay vectors of a single scalar function of $\mathbf{x}, y(t)=h(\mathbf{x}(t))$, under rather general conditions. The mapping,

$$
\Phi(\mathbf{x}(t))=(y(t), y(t+\tau), \ldots, y(t+(m-1) \tau)),
$$

which maps $\mathbf{x}$ to an $m$-dimensional delay vector with delay $\tau$, is an embedding when $m \geqslant 2 n+1$. This means that most of the significant characteristics of the original system, both dynamical and geometrical, are carried over to the reconstructed phase space in a one-to-one manner. ${ }^{30,38}$ In particular, properties such as the fractal dimension, Lyapunov exponents, and entropies are preserved under the reconstruction map $\Phi$ and can be computed from the mirror dynamical flow in the reconstructed space. There exist further generalizations that serve to reduce the bound on the embedding

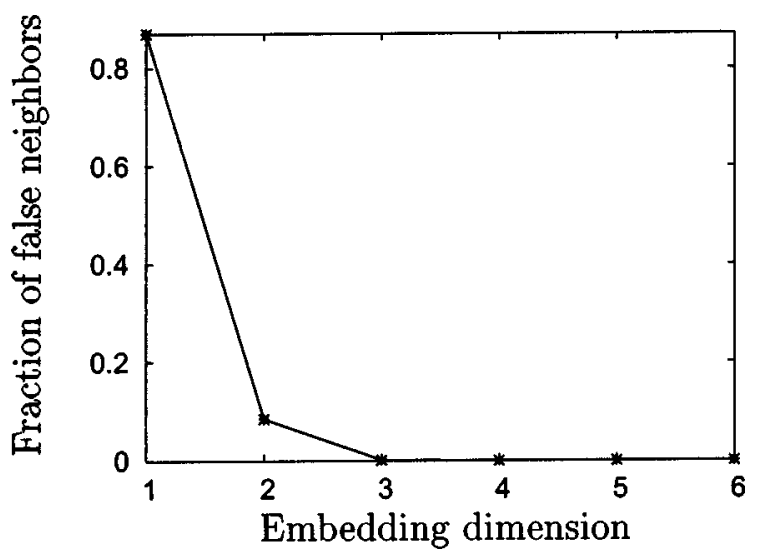

FIG. 5. Fraction of false nearest neighbors as a function of the embedding dimension $m$ for the $\left[\eta_{2}\right]$ series.

dimension, and in many cases the smallest integer greater than the correlation dimension is enough to fully embed the attractor. ${ }^{36,37}$

Although the embedding theorems do not place any restriction on the choice of the time delay, in practice, the choice of both the time delay and embedding dimension is important and may significantly affect the inferences derived from reconstruction, particularly when the data come from experiment. Small delays lead to highly correlated vectors $\Phi(\mathbf{x}(t))$, while large delays yield vectors with more or less uncorrelated components resulting in data randomly distributed in the embedding space. A first guess of the proper choice of the delay may be obtained from the autocorrelation function of the sample data; the time at which the autocorrelation attains its first zero, or its first local minimum, can be taken as the optimal delay. ${ }^{30}$ For our time series this value was around $\tau=15$ and we got topologically identical attractors for other choices of delay around this value. As for the embedding dimension $m$, it should be large enough for the attractor to fully unfold in the embedding space, but choosing too large an $m$ may cause the various algorithms to underperform. ${ }^{30} \mathrm{~A}$ commonly used method to estimate the optimal value of $m$ is the false nearest neighbor method, ${ }^{39,40}$ which is based on the idea that a small value for $m$ would not unfold the true geometry of the attractor and there may be self-intersections leading to false neighbors. Figure 5 plots the fraction of false neighbors as a function of the embedding dimension $m$ and yields $m=3$ as an optimal choice, since for $m \geqslant 3$ the fraction of false neighbors become very small. This means that the behavior of the system can be eventually described by utmost three, independent coordinates. Figure 6 shows the attractor reconstructed from the time series of $\left[\eta_{2}\right]$ with $m=3$ and $\tau=15$. We experimented with higher dimensions and various delays, but in all cases the attractor was found to be topologically identical to the one in the figure. We note that there is a definite structure in the phase phase plot of the stress component.

A quantitative measure of the structure and selfsimilarity of the attractor is provided by various dimension estimates such as the box-counting dimension, the Hausdorff dimension, etc. The correlation dimension, introduced by Grassberger, ${ }^{41}$ Grassberger and Procaccia, ${ }^{42}$ and others, is the 


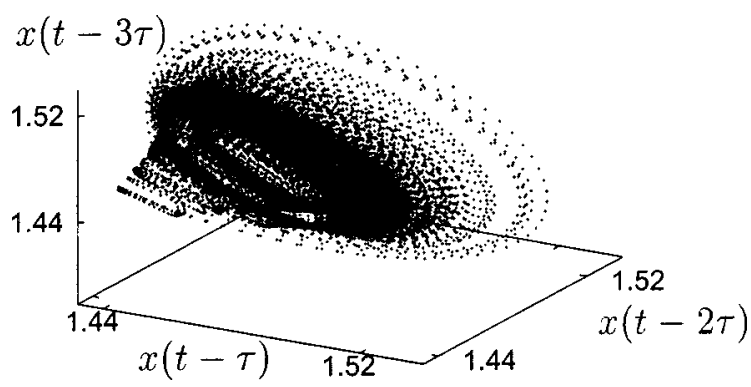

FIG. 6. Three-dimensional embedding of the attractor of $\left[\eta_{2}\right]$ reconstructed from the time series with delay 15 and $m=3$.

easiest to compute from a time series. The correlation dimension is estimated from the correlation sum $C(\epsilon, N)$, which is defined as the fraction of all possible pairs of points in the attractor, which are closer than a given distance $\epsilon$ in a given norm $;^{30}$

$$
C(\epsilon, m)=\frac{2}{N(N-1)} \sum_{i=1}^{N} \sum_{j=i+1}^{N} \Theta\left(\epsilon-\left\|\mathbf{x}_{i}-\mathbf{x}_{j}\right\|\right),
$$

where $\Theta(x)=1$ if $x>0, \Theta(x)=0$ if $x \leqslant 0$ and $\mathbf{x}(t)$ is the $m$-dimensional vector of time-delay coordinates. The correlation dimension is then given by

$$
D=\lim _{\epsilon \rightarrow 0} \frac{\partial \ln C(\epsilon, m)}{\partial \ln \epsilon},
$$

when $m$ is sufficiently large. The scaling exponent $\ln C(\epsilon, m) / \ln \epsilon$ typically increases with $m$ and saturates to a final value for sufficiently large $m$, which is then taken as an estimate for $D$. In practice, the values $\ln C(\epsilon, m) / \ln \epsilon$ are plotted against $\epsilon$ for various $m$ and the value corresponding to a plateau in the curves is identified as an approximation to $D$. In calculations, however, one has to be careful that the sum in Eq. (11) is not biased by temporal correlations, that is, the spatial closeness of the points appearing in Eq. (11) is not due to their being temporally close. ${ }^{43}$ This is done by excluding from Eq. (11) the pairs of points that are closer in time by less than a Theiler window, which is approximately equal to the product of the time lag between the points and the embedding dimension. ${ }^{43}$ In our calculations we used 50 as a Theiler window. Figure 7 plots the correlation sums $C(\epsilon, m)$ obtained with these choice of parameters, which shows a convergence of the curves for larger $m$, an indication of low

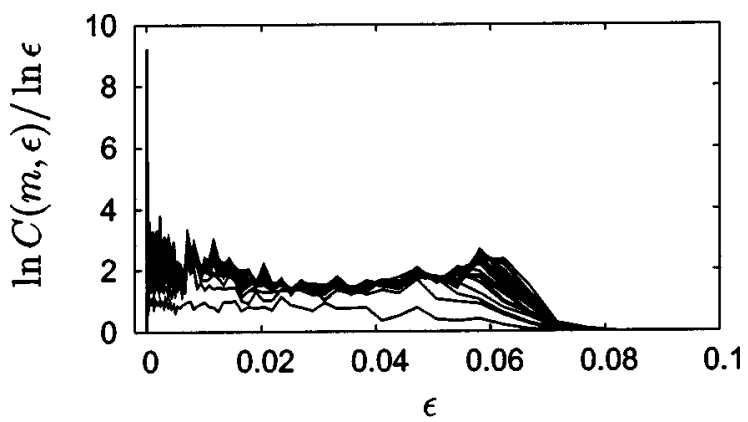

FIG. 7. Plot of $\ln C(m, \epsilon) / \ln \epsilon$ vs $\epsilon$. The convergence of the curves for large $m$ indicates low dimensionality.

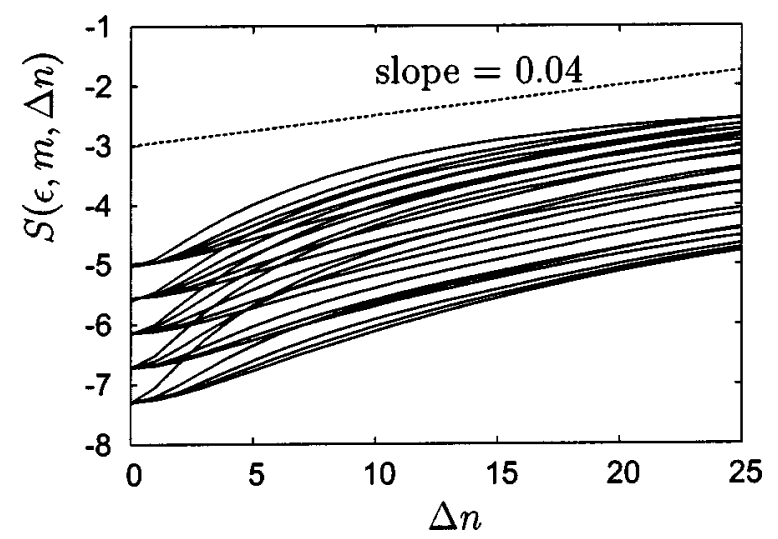

FIG. 8. The functions $S(\epsilon, m, \Delta n)$ vs $\Delta n$ for various embedding dimensions. The curves are approximately linear with an overall slope of 0.04 .

dimensionality of the attractor, and a plateau for the scaling exponent in the range $0.2 \leqslant \epsilon \leqslant 0.6$, suggesting a dimension equal to 2, approximately. Together with the presence of a definite structure in the attractor, this indicates that the apparent dimension of the system, governed by a set of 100 pairs of simultaneous equations, is far less than the number of degrees of freedom.

An interesting feature of some dynamical systems is their sensitive dependence on initial conditions, meaning that trajectories that start from neighboring initial conditions may diverge exponentially over time. An aperiodic bounded system having this property is termed a chaotic system. The Lyapunov exponents quantify the average rate of divergence or convergence of nearby orbits, and the existence of a positive Lyapunov exponent is one of the most striking signatures of chaos. ${ }^{32,44}$ Lyapunov exponents describe the longterm behavior of nearby trajectories and are invariant under smooth transformations of the attractor; hence they are preserved under delay reconstruction. We used the Kantz algorithm $\mathrm{m}^{30,45}$ to estimate the maximum Lyapunov exponent. This proceeds by computing the sum

$S(\epsilon, m, \Delta n)$

$$
=\frac{1}{N} \sum_{n_{0}=1}^{N} \ln \left(\frac{1}{\mid U\left(\mathbf{x}_{\mathbf{n}_{\mathbf{0}}}\right)} \sum_{x_{n} \in U\left(x_{n_{0}}\right)}\left|\mathbf{x}_{n_{0}+\Delta n}-\mathbf{x}_{n_{0}+\Delta n}\right|\right),
$$

for a point $\mathbf{x}_{n_{0}}$ of the time series in the embedded space and over a neighborhood $U\left(\mathbf{x}_{n_{0}}\right)$ of $\mathbf{x}_{n_{0}}$ with diameter $\epsilon$. If the plot of $S(\epsilon, m, \Delta n)$ against $\Delta n$ is linear over small $\Delta n$ and for a reasonable range of $\epsilon$, and all have an identical slope for sufficiently large values of the embedding dimension $m$, then that slope can be taken as an estimate of the maximum Lyapunov exponent. ${ }^{30}$ For our time series, Fig. 8 shows that $S(\epsilon, m, \Delta n)$ increases linearly with $\Delta n$ and that the slope is roughly independent of the embedding dimension $m$ for large $m$. An approximate estimate for the maximum Lyapunov exponent as obtained from the figure is 0.04 . These evidences show that the dynamics of the stress component is (weakly) chaotic in the range of parameters considered and that the system has a low-dimensional chaotic attractor in this case.

The response of the system to variations in the strength of the force field, with the field orientation kept unchanged, 


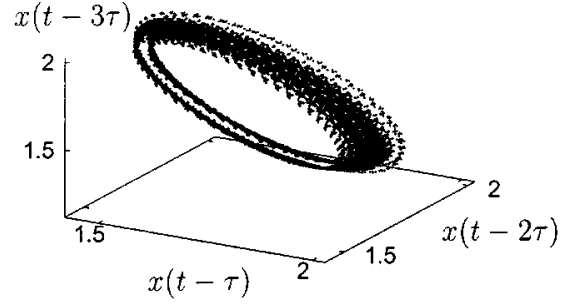

(a)

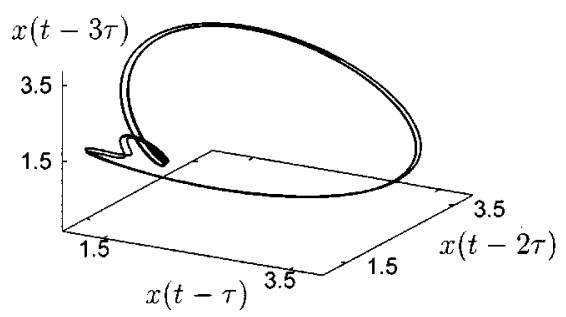

(c)

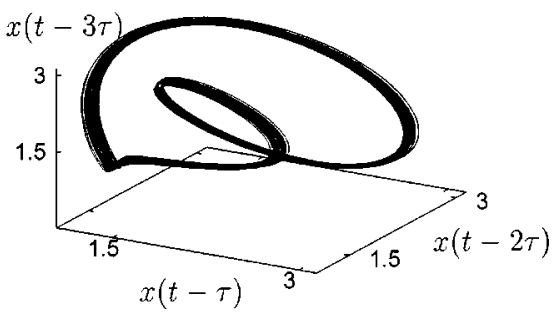

(b)

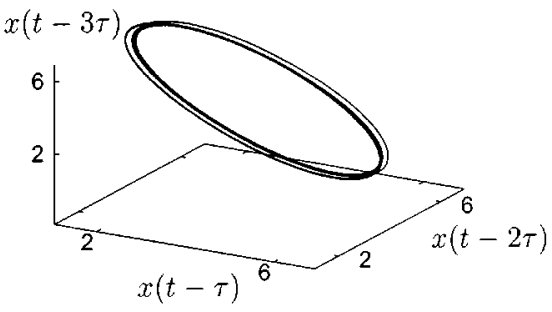

(d)
FIG. 9. Three-dimensional embeddings of the attractor reconstructed from the time series of $\left[\eta_{2}\right]$ for Pe $=0.01, k_{1}=k_{3}=0$, and various values of $k_{2}$; (a) $k_{2}=0.3$, (b) $k_{2}=0.42$, (c) $k_{2}=0.5$, and (d) $k_{2}=1.0$. are plotted in Figs. 9(a)-9(d). Shown in the figures are the three-dimensional embeddings of the attractor of the system for certain increasing values of $k_{2}$, viz., $k_{2}=0.3,0.42,0.5$, and 1.0, respectively. For other values of $k_{2}$ in the range we got attractors that are topologically identical to the ones in the figures. This suggests that the system takes a quasiperiodic route to chaos as the parameter $k_{2}$ is decreased from 1.0 to 0.1 .

The flow parameter $\dot{\gamma}$ tends to drive the particle distribution to an anisotropic state, which is either complemented or opposed by the interaction of the imposed force field, and the interplay between these forces can lead to chaotic fluctuations, both in the dynamics and in the rheology, in the absence of diffusion. ${ }^{17,18}$ The Brownian parameter $D_{r}$ has a smoothing effect on the distribution and tends to drive the system to an isotropic equilibrium. It is, therefore, interesting to observe that the bulk system response can be chaotic in the weak diffusion regime also, as we have demonstrated above, and we expect the system to revert to regular behavior when the diffusion gets stronger. This is illustrated by Figs. 10(a)-10(c), plotting the three-dimensional embeddings of the attractors corresponding to $\widetilde{\mathrm{P}}=0.0,0.1$, and 1.0 .

We note that all the above results pertain to the region of small Brownian motion, where the solution of the FokkerPlanck equation becomes otherwise intractable. In the limit of weak diffusion, the diffusion equation changes from a second-order partial differential equation to a first-order partial differential equation and the fundamental character of the equation changes from a diffusion equation type to a Liouville type. In perturbation methods, this often leads to a breakdown of the regular perturbation and requires a singular perturbation to be used. This usually means sharp gradients in the solution that are normally difficult to handle. The Langevin equation method is more preferable in this case

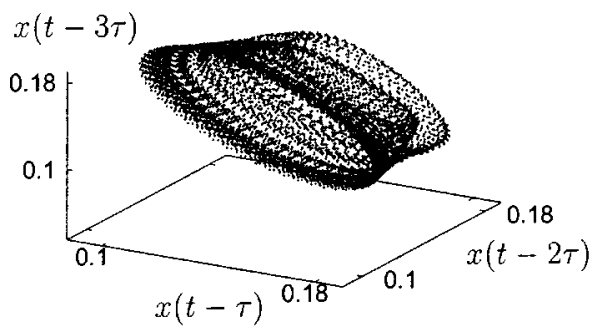

(a)

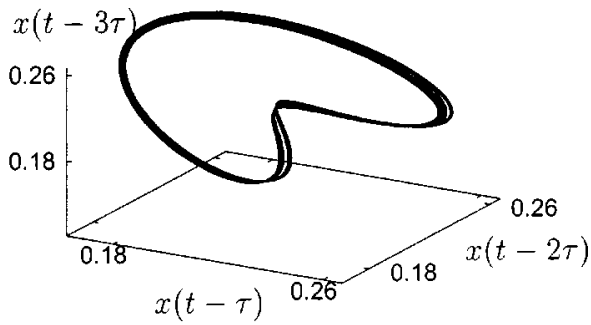

(b)

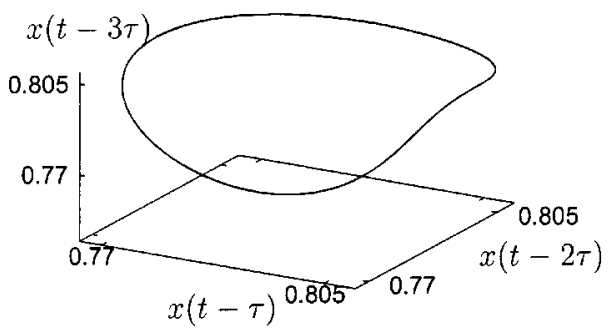

FIG. 10. Three-dimensional embeddings of the attractor reconstructed from the time series of $\left[\eta_{2}\right]$ for $k_{2}$ $=0.1, k_{1}=k_{3}=0$, and various values of $\mathrm{Pe}$; (a) $\mathrm{Pe}=0.0$, (b) $\mathrm{Pe}=0.1$, and (c) $\widetilde{\mathrm{P}} \mathrm{e}=1.0$.

(c) 


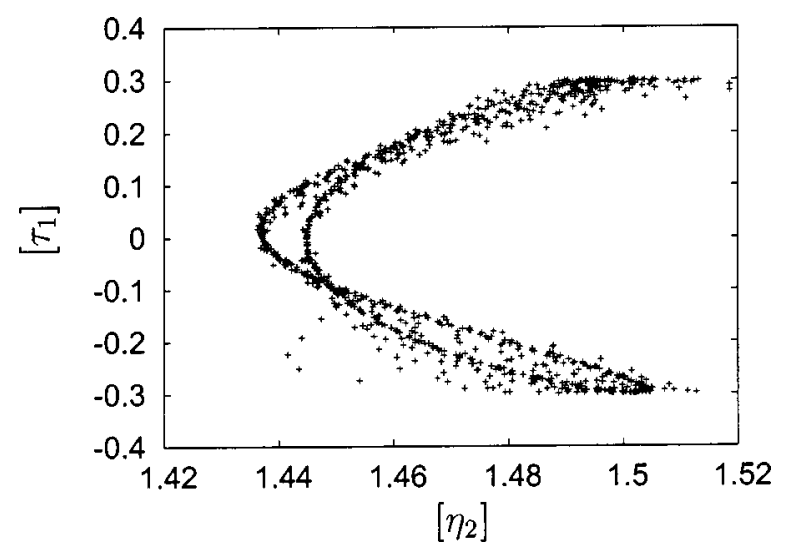

FIG. 11. Poincare section of the attractor of $\left[\eta_{2}\right] \times\left[\tau_{1}\right]$ for $\widetilde{\mathrm{P}} \mathrm{e}=0.01, k_{2}$ $=0.1, k_{1}=k_{3}=0$.

since, together with the paired-moment scheme for generating moments, it works well even when diffusion is weak and is capable of capturing possible complex behaviors of the system which methods based on singular perturbations may not be able to pick up.

Another important point to note is that the chaotic behavior of the rheological parameters could not have been picked up by many of the diffusion equation approaches that have been used to solve similar problems in the literature, either due to the deficiency of the approximation schemes employed to solve the diffusion equation or possibly due to more fundamental problems. Strand and Kim, ${ }^{4}$ for example, used an expansion of the ODF into a series of spherical harmonics and applied the Galerkin method to an appropriately truncated series to express the rheological parameters in terms of the expansion coefficients. Strand ${ }^{46}$ has applied this method to treat periodically forced systems of dipolar particle suspensions. Their expansion for the ODF permits only the driving frequency and higher harmonics of the shear and external field and is generally not valid in regimes where the stress fluctuations may have subharmonic periodicity, such as the chaotic parameter regimes we have explored, where the range of the frequencies is a continuum. Thus, if we take the Poincare sections of the time series $\left[\eta_{2}\right]$ vs $\left[\tau_{1}\right]$, i.e., snapshots of the attractor $\left[\eta_{2}\right] \times\left[\tau_{1}\right]$ taken at regular time intervals corresponding to the driving frequency, the method of Strand should give only a single point, whereas our method results in a continuum of points for the set of parameters considered above (Fig. 11). Thus, the existence of chaos in the region of weak Brownian motion adds another dimension to the computational difficulty of the problem with respect to the diffusion equation approach.

\section{CONCLUSIONS}

We have developed the Langevin equations for the orientations of dipolar particles in a simple shear flow subject to an external force field. Using an appropriate time-averaged form of these equations various rheological parameters were computed for different strengths of the diffusion and force field. The fluctuations in the apparent viscosity have been found to be chaotic for a set of parameters corresponding to weak diffusion when the external force is periodic, but a dominant Brownian motion or stronger force field tend to drive the system to regular behavior. The attractor in this case is low dimensional, showing that the system can be eventually described by a few independent coordinates.

One advantage of the method presented in this paper is that it picks up possible complexities in the dynamics of the bulk properties, such as the existence of subharmonic frequencies or sensitive dependence of the system behavior on initial conditions, which may not be identifiable in the diffusion equation approach. The important implication of this observation is that systems which are in some sense governed by the Fokker-Planck equation can show chaos in averages, and hence this system could become an important physically realizable system to determine the conditions under which chaos in microscopic dynamics can result in chaos in macroscopic averages. It can also be applied to a wider range of system parameters, unlike in the diffusion equation method, which requires, for better accuracy, a change in the strategy as the parameters are changed. ${ }^{23}$ It is also possible to extend the technique to more general systems, e.g., the suspension of charged fibers, ${ }^{47}$ which requires only an appropriate modification to Eq. (3).

\section{ACKNOWLEDGMENTS}

The authors thank Dr. B. C. Pai, Director, RRL, for constant encouragement. They also thank Dr. C. S. Bhat, J. Dasan, K. Radhakrishnan, and Tito Paul for useful discussions. The help rendered by V. Hema in the preparation of the manuscript is acknowledged. One of the authors (K.A.) wishes to acknowledge CSIR, India, for financial help during the initial stage of this work.

${ }^{1}$ Yu. A. Buevich, S. V. Syutkin, and V. V. Tetyukhin, "Theory of a developed magnetofluidized bed," Magnetohydrodynamics (N.Y.) 20, 333 (1984).

${ }^{2}$ N. M. Ignatenko, Yu. Melik-Gaikazyan, V. M. Polunin, and A. O. Tsebers, "Excitation of ultrasonic vibrations in a suspension of uniaxial ferromagnetic particles by volume magnetostriction," Magnetohydrodynamics (N.Y.) 20, 237 (1984).

${ }^{3}$ A. Cebers, "Chaos: New trend of magnetic fluid research," J. Magn. Magn. Mater. 122, 281 (1993).

${ }^{4}$ S. R. Strand and S. Kim, "Dynamics and rheology of a dilute suspension of dipolar non-spherical particles in an external field: Part I. Steady shear flows," Rheol. Acta 31, 94 (1991).

${ }^{5}$ R. E. Rosenweig, Ferrohydrodynamics (Cambridge University Press, New York, 1985).

${ }^{6}$ T. J. Pedley and J. O. Kessler, "A new continuum model for suspension of gyrotactic micro-organisms," J. Fluid Mech. 122, 155 (1990).

${ }^{7}$ T. J. Pedley and J. O. Kessler, "Hydrodynamic phenomena in suspensions of swimming micro-organisms," Annu. Rev. Fluid Mech. 24, 313 (1992).

${ }^{8}$ W. F. Hall and S. N. Busenberg, "Viscosity of magnetic suspensions," J. Chem. Phys. 51, 137 (1969).

${ }^{9} \mathrm{H}$. Brenner, "Rheology of a dilute suspension of dipoar spherical particles in an external field," J. Colloid Interface Sci. 42, 141 (1970).

${ }^{10} \mathrm{H}$. Brenner and M. H. Weissman, "Rheology of a dilute suspension of dipolar spherical particles in an external field. II. Effect of rotary Brownian motion," J. Colloid Interface Sci. 41, 499 (1972).

${ }^{11} \mathrm{~K}$. M. Jansons, "Determination of the constitutive equations for a magnetic field," J. Fluid Mech. 137, 187 (1983).

${ }^{12}$ C. Salueña, A. Pérez-Madrid, and J. M. Rub'i, "The viscosity of a suspension of rod-like particles,” J. Colloid Interface Sci. 164, 269 (1994).

${ }^{13}$ T. L. Smith and C. A. Bruce, "Intrinsic viscosities and other rheological properties of flocculated suspensions of nonmagnetic and magnetic ferric oxides," J. Colloid Interface Sci. 72, 13 (1979). 
${ }^{14}$ Y. Almog and I. Frankel, "The motion of axisymmetric dipolar particles in homogeneous shear flow," J. Fluid Mech. 289, 243 (1995).

${ }^{15}$ Y. Almog and I. Frankel, "Rheology of dilute suspensions of Brownian dipolar axisymmetric particles," J. Fluid Mech. 266, 289 (1998).

${ }^{16}$ T. R. Ramamohan, S. Savithri, R. Sreenivasan, and C. C. S. Bhat, "Chaotic dynamics of a periodically forced slender body in a simple shear flow," Phys. Lett. A 190, 273 (1994).

${ }^{17}$ C. V. Kumar, K. S. Kumar, and T. R. Ramamohan, "Chaotic dynamics of periodically forced spheroids in simple shear flow with potential application to particle separation," Rheol. Acta 34, 504 (1995).

${ }^{18}$ K. S. Kumar and T. R. Ramamohan, "Chaotic rheological parameters of periodically forced slender rods in simple shear flow," J. Rheol. 39, 1229 (1995).

${ }^{19}$ K. S. Kumar, S. Savithri, and T. R. Ramamohan, "Chaotic dynamics and rheology of suspensions of periodically forced slender rods in simple shear flow," Jpn. J. Appl. Phys. 35, 5901 (1996).

${ }^{20} \mathrm{C}$. V. Kumar and T. R. Ramamohan, "New Class I intermittency in the dynamics of periodically forced spheroids in simple shear flow," Phys. Lett. A 227, 72 (1997).

${ }^{21}$ C. V. Kumar and T. R. Ramamohan, "Controlling chaotic dynamics of periodically forced spheroids in simple shear flow: Results for an example of a potential application," Sadhana: Proc., Indian Acad. Sci. 23, 131 (1998).

${ }^{22}$ K. Radhakrishnan, K. Asokan, J. Dasan, C. C. Bhat, and T. R. Ramamohan, "Numerical evidence for the existence of a low-dimensional attractor and its implications in the rheology of dilute suspensions of periodically forced slender bodies," Phys. Rev. E 60, 6602 (1999).

${ }^{23}$ K. Asokan, T. R. Ramamohan, and V. Kumaran, "A novel approach to computing the orientation moments of spheroids in simple shear flow at arbitrary Péclet number," Phys. Fluids 14, 75 (2002).

${ }^{24}$ W. T. Coffey, Yu. P. Kalmykov, and J. T. Waldron, The Langevin Equation (World Scientific, Singapore, 1996).

${ }^{25}$ W. H. Press, B. P. Flannery, S. A. Teukolsky, and W. T. Vetterling, $\mathrm{Nu}-$ merical Recipes, The Art of Scientific Computing (Cambridge University Press, Cambridge, 1986).

${ }^{26}$ A. Okagawa and S. G. Mason, "Particle behavior in shear and electric fields: VII. Orientation distribution of cylinders," J. Colloid Interface Sci. 47, 568 (1974).

${ }^{27}$ J. P. McTague, "Magnetoviscosity of magnetic colloids," J. Chem. Phys. 51, 133 (1969).

${ }^{28}$ K. Sudou, Y. Tomita, R. Yamane, Y. Ishibashi, and H. Otowa, "Ferromag- netic fluid flow through a circular channel," Bull. JSME 26, 2120 (1983).

${ }^{29} \mathrm{~T}$. Weser and K. Stierstadt, "Magnetoviscosity of concentrated ferrofluids," Z. Phys. B: Condens. Matter 59, 257 (1985).

${ }^{30}$ H. Kantz and T. Schreiber, Nonlinear Time Series Analysis (Cambridge University Press, Cambridge, 1997).

${ }^{31}$ R. Hegger, H. Kantz, and T. Schreiber, "Practical implementation of nonlinear time series methods: The TISEAN package," Chaos 9, 413 (1999).

${ }^{32}$ H. Schuster, Deterministic Chaos (Physik-Verlag, Weinheim, Germany, 1988).

${ }^{33}$ A. Tsonis, Chaos: From Theory to Application (Plenum, New York, 1992).

${ }^{34}$ N. H. Packard, J. P. Crutchfield, J. D. Farmer, and R. S. Shaw, "Geometry from a time series," Phys. Rev. Lett. 45, 712 (1980).

${ }^{35} \mathrm{~F}$. Takens, Detecting Strange Attractors in Turbulence, Lecture Notes in Math ematics (Springer, New York, 1981), Vol. 898.

${ }^{36}$ T. Sauer, J. A. Yorke, and M. Casdagli, “Embedology,” J. Stat. Phys. 65, 579 (1991).

${ }^{37}$ T. Sauer and J. A. Yorke, "How many delay coordinates do you need?" Int. J. Bifurcation Chaos Appl. Sci. Eng. 3, 737 (1993).

${ }^{38}$ E. Ott, T. Sauer, and J. A. Yorke, Coping with Chaos (Wiley, New York, 1994).

${ }^{39}$ M. B. Kennel, R. Brown, and H. D. I. Abarbanel, "Determining embedding dimension for phase-space reconstruction using a geometrical construction," Phys. Rev. A 45, 3403 (1992).

${ }^{40}$ H. D. I. Abarbanel, Analysis of Observed Chaotic Data (Springer-Verlag, Berlin, 1996).

${ }^{41}$ P. Grassberger, "Generalized dimension of strange attractors," Phys. Lett. A 97, 227 (1983).

${ }^{42} \mathrm{P}$. Grassberger and I. Procaccia, "Measuring the strangeness of strange attractors," Physica D 9, 189 (1983).

${ }^{43}$ J. Theiler, "Spurious dimension from correlation algorithms applied to limited time series data," Phys. Rev. A 34, 2427 (1986).

${ }^{44}$ E. Ott, Chaos in Dynamical Systems (Cambridge University Press, Cambridge, 1993).

${ }^{45} \mathrm{H}$. Kantz, "A robust method to estimate the maximal Lyapunov exponent of a time series," Phys. Lett. A 185, 77 (1994).

${ }^{46}$ S. R. Strand, "Dynamic rheological and rheo-optical properties of dilute suspensions of dipolar Brownian particles," Ph.D. thesis, University of Wiscosin, Madison, 1989.

${ }^{47}$ S. B. Chen and D. L. Koch, "Rheology of dilute suspensions of charged fibers," Phys. Fluids 8, 2792 (1996). 